\title{
CHAPTER 11B
}

\section{COMMENTS ON "STRUCTURING HERBIVORE COMMUNITIES: THE ROLE OF HABITAT AND DIET”}

\author{
HERBERT H.T. PRINS, AREND M.H. BRUNSTING AND \\ WILLEM F. DE BOER \\ Resource Ecology Group, Wageningen University, P.O. Box 47, \\ 6700 AA Wageningen, The Netherlands \\ E-mail: herbert.prins@wur.nl
}

The question Van Wieren and Van Langevelde (Chapter 11) are trying to find an answer to, namely "Why are there so many species?", and especially "Why are there so many herbivore species at some location?" is an intriguing one, but not a simple one. To solve this question, one must first look into the exact articulation of the question. The word "Why" is particularly knotty. The question could be reformulated as "What causes the existence of so many species?", but also as "What is the function of so many species?" or even "How did so many species evolve?”. At first sight, Van Wieren and Van Langevelde deal with the first question, about the cause. However, a closer look at the text reveals that they try to find an answer to another question than the one they pose, namely, "What allows so many different species to co-exist?”. This is a pity, because if they had tried to find an answer to the question about causality, then they would have taken, hopefully, an evolutionary and dynamic approach. Now their approach is static, and focuses on the conditionality instead of the causality.

In their search for conditionalities of co-existence, they justifiably concentrate on herbivores, and especially African herbivores, because of the superb species richness of this group. They then tackle their question armed with niche theory. The 'niche' concept is fraud with difficulties, and they rely heavily on Chase and Leibold (2003). It was good to see that they do refer back to the early meanings of the word 'niche' by referring to Grinnell (1917) and Elton (1927); even today, a dictionary gives both sets of meanings ('place, position, slot, alcove, nook' reflecting the Grinnellian point of view versus 'function, role', which is the Eltonian one). Van Wieren and Van Langevelde then follow Chase and Leibold's (2003) new definition of 'niche' as "the joint description of the environmental conditions that allow a

263

Herbert H.T. Prins, Frank van Langevelde (eds.), Resource Ecology: Spatial and Temporal Dynamics of Foraging, 263-266.

(C) 2008 Springer. 
species to satisfy its minimum requirements so that the birth rate of a local population is equal to or greater than its death rate along with the set of per capita effects of that species on these environmental conditions" (the latter part of the definition is especially of importance for herbivores that can modify their resources). But, do they do so?

Their apportioning of the component species from an exemplar assemblage, that of Kruger National Park, over a set of niches is very Grinellian! Every species is allocated a place within the system based on its location (and not on its function), which tells little to nothing about the above-mentioned "minimum requirements so that the birth rate of a local population is equal to or greater than its death rate". The same is the case for assigning the species to diet classes. A combination of the two may be a first step leading to a Hutchinsonian $n$-dimensional hyperspace (Hutchinson 1957), but it is not a step towards solving the question of species richness. Indeed, one must seriously question whether diet class, just as body mass, is a proper niche parameter! Diet selection is the resultant from the interface between evolutionary derived predisposition and the local flora (possibly modified by the impact of other herbivores), while body mass is the result of evolution alone and a characteristic of the animal, not of its niche. There are in our view more problems with their analysis. We think that a niche dimension should be defined independently of the species making use of it. So this is another reason why diet is a dicey niche dimension, because it is not independently defined. They should have stuck to quantity and quality parameters, like they did so well at the beginning of the chapter, and on which especially Van Wieren is a rare specialist. Van Wieren and Van Langevelde use another niche dimension, namely 'habitat', to separate the different species of the Kruger assemblage. For that they chose to use the habitat classification of Table 11.2. In a strict Hutchinsonian sense, their eight categories do not lie on one dimension though, but they lie on 4 dimensions, namely grass cover, shrub cover, tree cover and woody species height (e.g., Loth and Prins 1986). Habitat thus does not form a niche dimension. However, strictly speaking, habitat is not a resource either, and a habitat category can thus not be an exclusive resource. Moreover, the habitat and diet classes used in their analysis are subjective, as one could also argue that both classes only comprise two major distinctions, namely forest versus grassland, or browse versus grass.

It thus appears as if Van Wieren and Van Langevelde (Chapter 11) have chosen to ignore the challenge they set themselves. Their chapter is neither addressing the question about why there are so many species, nor have they met the challenge put forward by the new definition of 'niche' by Chase and Leibold (2003). Their approach seems ad hoc, trying to find dimensions that separate the species and can explain the co-existence. This concept lacks rigorous scientific testing and null hypotheses. From their descriptive approach no conclusions can therefore be drawn, at best hypotheses could be formulated that could direct future research.

But even if habitat the way they use it could be considered a niche axis along which species have to find a place, how then does one explain the next issue of concern? If one studies Table 11.3 then the following emerges. On average a species makes use of more than one habitat (namely, of 2.7); elephant and buffalo are very catholic and use 8 and 5 habitats, respectively, and if we leave these two species out, 
the average species utilizes 2.5 habitats. This one would not have expected: one would have anticipated that species would be mutually exclusive due to competitive exclusion (which can only be studied of course by looking at the dynamics of the system). A finer subdivision of the 'niche space', as done in Table 11.9, does not help. If one leaves body mass out (because it is not a niche dimension), then the average number of species in one combinational category of 'habitat' and 'diet class' is 2.1 species (with a maximum of 7 species in the combination of 'variable grazers' and 'light savanna'). So, 7 African herbivore species in one locality can share a 'niche'. Finally, if one wants to be very generous, and if one would accept that body mass could be equated with a niche dimension, even then in Table 11.9 the maximum number of species sharing a combinational category of 'habitat', 'diet class' and 'body mass class' is 1.4 species, but with still maximally 3 species in a 'unique' combination (i.e., small-sized browsers in thickets: common duiker, suni and bushbuck, medium-sized variable grazers in a light savanna: warthog, hartebeest and waterbuck, the same combination in a woodland savanna, or large-sized variable grazers in such a savanna: wildebeest, buffalo and hippo, and finally, large-sized variable grazers in a bush savanna: sable, wildebeest and roan antelope). The hypothesis of Van Wieren and Van Langevelde that "a species can only exist if it has exclusive access to resources that cannot be used by others" is thus not supported by their own ordering and grouping of species, and more importantly, the justification behind the hypothesis is lacking, as is the hypothesis testing. The resulting categories simply do not equate to niches, and their categorization does not lead to the identification of resource dimensions.

When Van Wieren and Van Langevelde write that "When body mass ... is being added to the habitat-diet niche space, the community becomes much more structured", then they are, in a way right: indeed, every individual species gets an increasingly larger chance to become uniquely circumscribed if there are more unique combinations of descriptive categorical axes. However, we do not think that they have approached the answer to their question any closer. The basis for our assertion is that they have not chosen a dynamic or evolutionary tack. A dynamic course would have been necessary to meet Chase and Leibold's (2003) new definition, and an evolutionary approach would be indicated by the fact that body mass (and to a lesser extent habitat choice or food adaptations) are the outcomes of a competition between species over time.

The allotment of species to a Hutchinsonian-type multi-dimensional hyperspace is reminiscent of mediaeval Italian noble families living in their tower-like townhouses. Every family had its own townhouse, but did this description explain why there were so many towers? Or why in one town there were 72 of these towers (in San Gimignano), and in another 170 (in Lucca, inclusive of church towers)? Of course not! A description of the number and locations of these towers gives a description of the structure of such an Italian town, but this does not yield an explanation. The enlightenment comes from the dynamics, the evolution: which family was able to protect its locality, what tower was torn down by a competing family, and between which neighbours could one settle? Competition and facilitation (Prins and Olff 1998) are key concepts here, and that is why Chase and Leibold (2003) focus on birth rate and death rates of local populations. 
The hypotheses and conclusions put forward in their chapter need to be reconsidered. It is not clear whether the hypothesis that species can only co-exist if they have exclusive resource access is merely a reformulation of Gause's axiom. For instance, spatial or temporal heterogeneity in resource abundance can also promote species co-existence of two species that exploit a single resource (Ritchie and Olff 1999; Chesson 2000a; 2000b). The hypothesis that heterogeneity increases the size range of species needs further thoughts: what is the basis for this hypothesis? How would we be able to test these hypotheses? Another problem is that it is still unclear how important body size is in determining niche dimensions. It is clear that our quest for the mechanisms that determine species co-existence is a long one, and can only be completed by way of careful formulation and testing of hypotheses.

We do not fully support the research agenda which Van Wieren and Van Langevelde propose at the end of their chapter, because it harkens back to the Grinnellian and Hutchinsonian definitions of niche. What we think is needed, over and above (or perhaps even in place of) what they propose, is controlled experiments in which they bring Chase and Leibold's (2003) new definition of niche to the test. What is thus needed is that for different herbivores of the same or different body mass and with the same or a different 'adaptive syndromes' (Van Wieren and Van Langevelde, under different sets of environmental conditions, the minimum requirements of these herbivores under which the birth rate is equal to or greater than their death rate are determined. Then at a next stage it has to be determined what the sets of per capita effects of these chosen species are on these experimental environmental conditions. Penultimately, this has to be coupled with a phylogenetic study to understand the evolution of niche use better, and also to understand the body mass development over geological time for the different species. Finally, this research agenda calls for a careful study of phenotypic plasticity of body mass (and associated body measurements such as incisor arc width) and character displacement of species living with different competitors in different assemblages over the whole geographic range of the component species. What we propose is thus a research agenda that may ultimately answer one of the holy grails of modern science, namely "What causes the (co-)existence of so many species?". 